\title{
Les pouvoirs de la musique polynésienne
}

\section{Richard Moyle}

Traducteur : Isabelle Schulte-Tenckhoff

\section{OpenEdition}

\section{Journals}

Édition électronique

URL : http://journals.openedition.org/ethnomusicologie/2389

ISSN : 2235-7688

\section{Éditeur}

ADEM - Ateliers d'ethnomusicologie

\section{Édition imprimée}

Date de publication : 1 janvier 1990

Pagination : 141-150

ISBN : 2-8257-0423-7

ISSN : 1662-372X

\section{Référence électronique}

Richard Moyle, "Les pouvoirs de la musique polynésienne », Cahiers d'ethnomusicologie [En ligne], 3 | 1990, mis en ligne le 15 octobre 2011, consulté le 21 avril 2019. URL : http://

journals.openedition.org/ethnomusicologie/2389 


\title{
LES POUVOIRS DE LA MUSIQUE POLYNÉSIENNE*
}

\author{
Richard Moyle
}

En Polynésie, la musique peut bien davantage qu'«apaiser le cœur sauvage». En de nombreux contextes sociaux (public et privé, sacré et profane, collectif et individuel), elle sert tantôt à manipuler des événements ou des états dans le monde naturel, tantôt à communiquer avec le monde surnaturel, à évoquer des sujets ordinairement interdits, à atteindre la quintessence de la perfection linguistique ou encore à élever son statut social.

Ces virtualités de la musique se fondent sur des croyances liées au pouvoir de la parole énoncée. Le discours chanté ou récité ouvre des voies de communication autrement inaccessibles, en particulier lorsqu'il est structuré conformément aux formules linguistiques reconnues (c'est-à-dire poétiques au sens le plus large du terme); ainsi confère-t-il aux commandements une intensité irrésistible à tout être humain ou surhumain. Anciennement à Hawaï, par exemple, la puissance de la parole énoncée était telle que même les lieux où certains chants étaient exécutés devenaient tabous (Kamakau, cité in Roberts 1926: 59).

Dans le monde naturel, les «états de devenir » et «d'être» ne sont intrinsèquement ni positifs ni négatifs; ils sont des sortes de matériaux bruts mis à la disposition des hommes en vue de satisfaire leurs besoins physiques ou d'assurer leur avancement social par l'exaltation de soi ou le dénigrement d'autrui. Investis d'une puissance neutre qui peut favoriser ou défavoriser leurs usagers, ces états se prêtent à être manipulés par ceux-là mêmes qui sont capables d'y accéder. Le moyen d'accès privilégié est le chant, d'une manière qui défie l'explication rationnelle.

Traduit de l'anglais par Isabelle Schulte-Tenckhoff. D'une manière générale, ce bref essai s'inspire des recherches et réflexions menées depuis vingt-cinq ans par l'auteur sur la société et la musique polynésiennes, notamment dans les îles Samoa, Tonga, Lau, Niue et Cook, ainsi qu'à Hawaï. 
Si la musique - qu'elle soit vocale, instrumentale ou une combinaison des deux - est investie de pouvoirs, on peut raisonnablement supposer l'existence de mécanismes visant à l'intégrer sans préjudice au système des hiérarchies sociales, en limitant le nombre d'individus habilités à l'employer. Dans les sociétés où l'autorité se fonde sur l'âge en tant qu'il représente la sagesse acquise par un cumul de connaissances, des révélations faites au hasard risquent de compromettre le processus de sélection qui est à la base de l'autorité et de déformer ainsi le tissu social tout entier. Un contrôle est donc assuré par la désignation des individus admissibles (prêtres, chefs de famille) ou encore par la révélation graduelle du savoir nécessaire. Plus spécifiquement:

- C'est l'interprète, homme ou femme, qui cherche à influencer les événements et les états dans la nature; il y parvient en minimisant la part individuelle de sa contribution et en se concentrant sur le pouvoir inhérent à la formule poétique: le plus souvent, le texte est d'origine ancienne et anonyme, et il faut s'y conformer absolument; des prescriptions semblables régissent le mode d'interprétation, qui doit être parfait. L'interprète agit au nom de ceux qui réclament ses services.

- C'est le compositeur, homme ou femme, qui cherche à influencer l'opinion sociale en maximisant son identité, soit en tant qu'individu, soit en s'identifiant au groupe des interprètes: le plus souvent, le texte est moderne et se réfère à une question d'actualité; l'identité du compositeur est connue, et elle est exprimée dans la poésie par l'emploi fréquent de pronoms de la première personne (par exemple: «viens dans mon village si tu veux en savoir plus»; «nous sommes les gens qui font le guet»); quant au mode d'interprétation, il est plus flexible: par exemple, le nombre de chanteurs ou d'instrumentistes, le tempo ou la hauteur peuvent varier. Ici l'interprète agit au nom du compositeur.

En vertu du dynamisme des systèmes polynésiens de stratification sociale (voir Goldman 1970: 8), obéissant à un jeu complexe de rangs individuels acquis ou attribués tant dans le domaine sacré que dans la vie profane, le statut précis d'une personne était à tout moment sujet aux forces opposées de la subjugation et de l'élévation. Dans le passé, le rôle de la musique consistait surtout à entretenir le système, étant donné que les individus de haut rang étaient honorés par le chant; l'efficacité de la musique dans ce domaine ressort clairement du fait que les chants d'éloge survivaient aux personnages auxquels ils avaient été destinés, comme c'était notamment le cas à Hawaï. Là où de tels chants sont encore interprétés de nos jours, la musique a acquis un rôle plutôt cérémoniel. En revanche, les chants de vitupération adressés à un individu étaient généralement éphémères et connus seulement dans une région délimitée; leur emprise sociale résultait de leur actualité. Dans la mesure où «entretien» équivaut à maintien du statu quo, l'intégration fréquente de la musique au rite, c'est-à-dire à une sphère aux structures presque aussi rigides, contribuait à un ralentissement général du processus de changement et, par là même, à l'entretien de la source du pouvoir célébré ou invoqué par le rite. 
Si, en Polynésie, les pouvoirs de la musique sur la transformation du système des statuts s'inscrivent, en effet, dans la société elle-même, on peut raisonnablement s'attendre à ce que les musiciens bénéficient de la reconnaissance sociale et qu'il existe aussi des moyens de limiter les abus. La reconnaissance sociale passe à travers l'attribution de titres honorifiques (comme celui de punake dans les îles Tonga), le remplacement partiel ou intégral des orateurs par de grands chœurs ou ensembles de danseurs qui interprètent tel ou tel chant dans un cadre cérémoniel, ainsi que des dons de richesses (nourriture, nattes, terres). Le principal obstacle aux abus dont la musique pouvait faire l'objet réside dans l'inversion d'un détail relevé plus haut: si le compositeur cherche à s'identifier au groupe qui interprète sa propre composition, il faut que son œuvre corresponde aux attentes de ce groupe ou qu'elle soit du moins approuvée par lui. Comme les chants représentent un moyen subtil de communication de la parole, c'est surtout le texte qui compte pour l'évaluation d'une composition. Idéalement, des éléments comme la justesse des images, le recours à des allusions historiques ou mythologiques, les rimes, les références indirectes habiles et l'humour doivent être combinés de manière à mettre en valeur un thème donné.

Dans de nombreuses régions, compte tenu de la rapidité avec laquelle un matériau aussi dense est généralement débité, il va de soi que les strophes sont répétées afin que le public soit en mesure d'en apprécier le contenu. En adjoignant la musique au texte, on veille, en outre, à ce que ses caractères sonores reflètent et, par là même, rehaussent le thème abordé. Aussi, dans les compositions destinées à la danse, l'aspect visuel renforce ce qui est essentiellement un acte verbal. Il est généralement admis que les talents de poète, de musicien ou de chorégraphe sont réservés à des spécialistes; et ceux qui sont capables de créer dans les trois domaines bénéficient d'une forte reconnaissance sociale, d'un prestige durable et de quelque récompense matérielle. Il apparaît donc que plus la valeur sociale attribuée aux compositions répondant aux attentes locales est grande, plus les normes gouvernant chaque élément de leur création sont formelles et exigeantes. A leur tour, ces normes reflètent un processus de sélection garantissant que l'espace au sommet n'est occupé que par une poignée de créateurs.

Un parallèle existe dans certaines formes de musique instrumentale. Dans le passé en Polynésie occidentale, et jusqu'à il y a deux générations en certains endroits, le jeu du tambour à fente était la prérogative exclusive des hommes, tant pour la transmission des codes rythmiques que pour l'accompagnement de types particuliers de danses.

L'idée que la parole est investie de pouvoir a été étendue de diverses manières à la musique instrumentale. Surtout en Polynésie occidentale, la guimbarde était utilisée jadis pour faire la cour. Derrière les sons apparemment anodins de son instrument, le prétendant rendait son message (par exemple, le lieu et l'heure d'un rendez-vous) audible et intelligible à la femme aimée, tout en le dissimulant aux autres auditeurs - et ce d'une façon que les sources publiées ne précisent pas. 
Il est possible d'isoler des éléments de la parole énoncée pour former des idiomes musicaux proprement dits. C'est un fait bien connu qu'en Polynésie, on pouvait jouer à la flûte la mélodie de tel ou tel chant - mélodie qui conservait d'ailleurs souvent le titre du chant originel. Et dans deux régions au moins (Polynésie française et Nouvelle-Zélande), un compromis est attesté pour le XIX ${ }^{\mathrm{e}}$ siècle: des flûtes et des voix sonnant à l'unisson. A Tahiti et dans les îles Cook, on extrait le rythme syllabique de la parole: là les tambours accompagnant la danse exécutent des séries uniques de formules rythmiques dont chaque composante est basée sur le rythme naturel d'une phrase parlée que les instrumentistes mémorisent avant de commencer à jouer. Ainsi le jeu de ces deux types d'instruments - flûte et tambour - représente-t-il une extension de la parole; par l'addition d'un champ de significations supplémentaire, le plaisir esthétique du public se trouve augmenté.

L'idée que le son est lui-même symbole et constitue de ce fait sa propre base de pouvoir (par exemple comme voix des ancêtres), est largement répandue en Mélanésie, mais peu attestée en Polynésie. Bien que les conques qui produisent de tels sons soient généralement qualifiées d'instruments de musique, elles n'entrent pas dans le champ présent de mes préoccupations.

En un sens, chaque fonction de la musique polynésienne peut être comprise comme un «pouvoir»: de communiquer, de divertir, de symboliser, et ainsi de suite; et ces fonctions sont nombreuses et variées (Merriam 1964: chap. 11). Pour mon propos, il est utile de les répartir selon qu'elles exercent un effet direct ou indirect sur les êtres humains: les premières visent à solliciter une réaction dans l'audience; les secondes s'accomplissent à travers un agent surhumain mobilisé au nom d'un requérant humain.

De toute évidence, la notion - largement répandue en Polynésie - d'une «essence vitale» contenue dans toute catégorie d'objet relève de l'animisme. Le protocole le plus strict est de rigueur lorsque la musique accompagne des événements directement ou indirectement liés à un changement d'état dans la vie humaine. L'essence vitale est déterminée culturellement dans la mesure où elle ne peut être atteinte qu'au moyen de certaines formules verbales énoncées dans l'idiome local. En outre, elle est déterminée par l'appartenance sociale, car seules certaines catégories de personnes ont le statut requis pour prononcer ces formules, de surcroît après un apprentissage et une expérience prolongés. La «magie des jardins", largement pratiquée en Mélanésie insulaire pour assurer de bonnes récoltes, n'est guère attestée en Polynésie, la plupart des exemples de ce type provenant de Nouvelle-Zélande.

La santé individuelle est l'état d'existence le plus communément influencé par l'intermédiaire de la musique. La plupart des changements souhaités sont induits par des traitements curatifs qui coexistent avec d'autres traitements impliquant l'application ou l'ingestion' de décoctions d'herbes et le massage de la zone corporelle affectée. Si l'idée de propriété associée aux préparations médicinales justifie le secret entourant leur fabrication, c'est la crainte du surnaturel qui explique le caractère secret des cures exigeant la pratique d'incantations. Pour invoquer et manipuler des forces puissantes mais invisibles, le guérisseur 


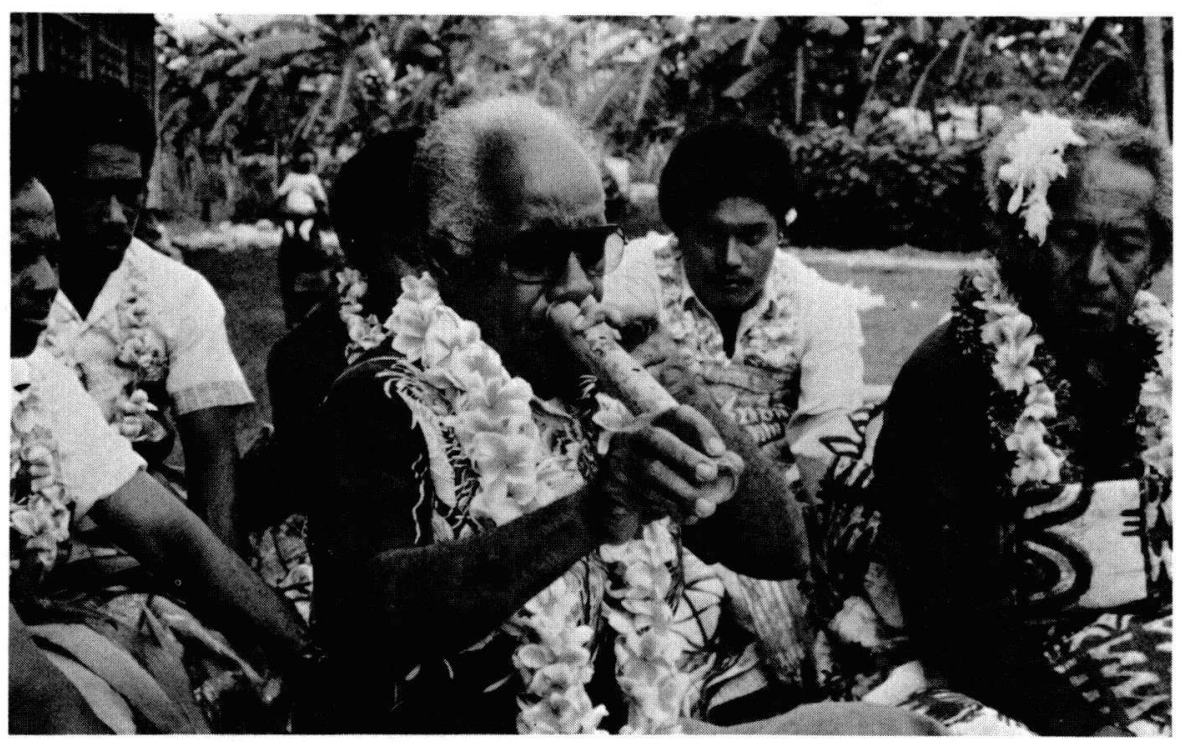

Fig. 1: Tonga: flûte nasale fangufangu dont la fonction d'instrument de musique récréative domestique a été largement supplantée par de la musique enregistrée. Photo: Habib H. Touma, 1988.

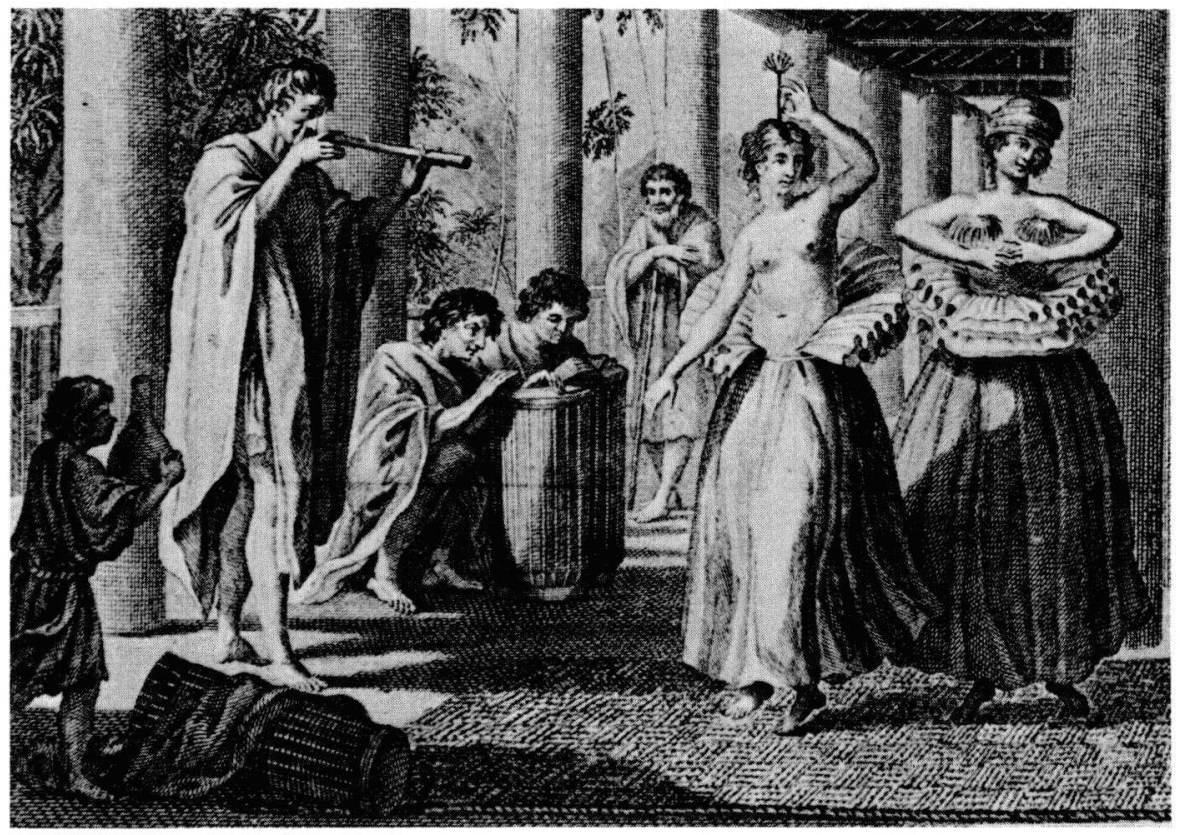

Fig. 2: Danseuses et musiciens jouant de la flûte nasale et du tambour dans l'île de Ulietéa (Iles de la Société). Tiré de: Atlas - 2 voyage de Cook, Paris: 1774: Tome III, pl. 6 (extrait). Légende originale: «Vue de l'intérieur d'une Maison dans l'Isle d'Ulietéa. Représentation d'une danse à la mode du pays.» Photo: J.-P. Dumontier. 
doit soigneusement cibler son intervention, au risque de contracter lui-même la maladie de son patient. Chez les Maori, la maladie était souvent attribuée à la violation d'un tapu, et sa guérison exigeait l'interprétation d'une certaine incantation au texte fixe mais néanmoins «ouvert», en ce sens qu'il ne se référait à aucune entité nommée ${ }^{1}$. En revanche, les incantations médicinales chantées ou scandées dans les îles Samoa désignent nommément l'esprit maléfique que l'on croit à l'origine de la maladie, car l'on pense que la guérison est assurée si l'on identifie correctement, dans les formules mêmes de l'incantation, la source du désordre. Or les Samoans n'en pratiquent pas moins une médecine empirique, car ils n'admettent avoir repéré l'esprit maléfique - et donc avoir réussi le traitement - qu'au moment où le patient est véritablement guéri: post hoc ergo propter hoc.

L'un des deux pouvoirs suprêmes attribués à la musique, à savoir de ressusciter les morts, est aussi attesté en Polynésie occidentale, mais seulement par les récits fictifs qui se racontent autour du foyer comme divertissement. Il est probable que le public local accepte sans autre les intrigues, en particulier les épisodes relatant une résurrection, parce que celles-ci se déroulent à la manière des traitements curatifs appliqués dans la vie réelle. Le scénario le plus répandu veut qu'une femme, le plus souvent l'épouse de la victime, entonne une brève incantation sur la dépouille de l'homme. Chaque fois que le chant est répété, une partie du corps (un doigt, un pied) se ranime; l'incantation continue jusqu'à ce que la guérison soit complète. Dans aucun de ces récits, la nature de la mort et les raisons du succès du traitement ne sont tirées au clair. Pour sa part, l'assistance ne questionne pas la logique ou la véracité de tels récits, même si celle-ci reste incomprise.

L'autre pouvoir suprême de la musique, à savoir d'infliger la mort au moyen de la sorcellerie, est attesté chez les Maori de Nouvelle-Zélande dont quelque 130 types d'incantations ont été rapportés (McLean \& Orbell 1975: 18). Étant donné que la plupart d'entre elles étaient liées à la guerre, elles sont maintenant tombées dans l'oubli, comme celles qui visent à rendre inefficaces les armes de l'ennemi, à saper son courage, à lui donner envie de dormir ou à appesantir ses jambes. Pour frapper de malheur un voleur, on entonnait des incantations punitives. Tous ces types d'incantations ont un trait commun: elles propulsent le pouvoir de la parole au-delà du seuil spatial de l'ouie humaine. Leur exécution n'exige pas l'invocation de tel ou tel être surnaturel; on croyait que les formules linguistiques de circonstance étaient intrinsèquement dotées d'une puissance que libérait l'acte d'énonciation, sans accorder d'importance à la distance spatiale.

D'autres pouvoirs de la musique en rapport avec les changements d'état ont été attestés, le plus souvent chez les Maori de Nouvelle-Zélande. McLean

De nombreuses transcriptions de textes, comme ceux publiés par Buck (1966: chap. 6), sont constitués de phrases dont la structure grammaticale ou le sujet ne s'écartent pas du langage quotidien; de telles phrases acquièrent donc un pouvoir supplémentaire par le fait qu'elles sont énoncées en des circonstances appropriées. 
et Orbell (1979: 19-20) relèvent, entre autres, l'existence d'incantations capables de calmer la mer, de causer des inondations, de fendre la pierre, de faire flétrir les arbres et d'invoquer le tonnerre. On ne connaît que peu de textes de telles incantations, mais il est néanmoins permis d'affirmer ceci: en dépit du fait que les Maori associaient des divinités particulières à la plupart des activités humaines et des espaces terrestres et marins, leurs prêtres se bornaient à invoquer le phénomène en tant que tel; conjointement au rite adéquat, la puissance inhérente à la parole suffisait pour obtenir le résultat escompté.

Un trait saillant du langage de la poésie musicale destinée à induire un changement d'état est l'allusion directe. Deux exemples suffiront pour illustrer ce phénomène. Le premier provient de Nouvelle-Zélande (Hiroa 1966: 492):

«Quelle était la nourriture entrée dans ton estomac,

Causant des troubles, causant des désordres,

Causant des gémissements?

Peut-être était-ce un chardon?

La nourriture entrée dans ton estomac,

Causant des troubles, causant des désordres,

Causant des gémissements.

Ô, Ô, Ô tu guériras

Ô tu guériras.»

Le second a été recueilli dans les îles Samoa (Moyle 1988: 79):

«Mal de tête, mal de tête,

Saute dans l'arbre $k i$,

Saute dans l'arbre $v i$,

Saute dans l'arbre fetau,

Reste perché sur le bananier,

Sauve-toi dans la forêt,

Cherche refuge en quelque endroit lointain,

Car je suis sur le point de te porter un coup de lance.»

Si l'on admet que la vertu de telles formules réside dans la parole ellemême, ces formules apparaissent comme des clefs rituelles dont le maniement correct ouvre des voies de communication avec les pouvoirs capables d'induire un changement d'état. Il est généralement admis que les pouvoirs surhumains, qu'ils soient nommément identifiés par l'incantation ou évoqués par une suite de mots désignant un objet ou un phénomène, sont disponibles et de ce fait réceptifs à l'influence humaine. Comme je l'ai déjà dit, l'effet conjugué de diverses contraintes sociales (tapu, crainte d'une erreur involontaire ou accidentelle dans l'exécution) et du mode d'interprétation requis (rapide et voilé, à voix basse), ainsi que la prépondérance de croyances locales relatives à la spécialisation religieuse, empêchent que les formules linguistiques soient utilisées sans discernement. Entourées d'une double couche de mystère, elles se soustraient ainsi à la curiosité d'autrui ou à un examen logique minutieux. 
En Polynésie, on pense que les êtres résidant dans les différents univers spirituels peuvent être abordés par la voie régulière (notamment lors des cérémonies établies) aussi bien qu'irrégulière (en cas d'urgence médicale ou de guerre). La mythologie polynésienne abonde en récits qui relatent des rencontres entre êtres humains et surhumains, des aventures impliquant les uns comme les autres, des visites d'humains dans le monde des esprits et viceversa. Parmi les qualités humaines que le public polynésien repère dans de tels récits figurent la politesse, la circonspection, l'énergie, la résolution, la bravoure et, occasionnellement, la ruse. Dans l'ensemble, ces qualités ne peuvent cependant pas être tenues pour un modèle de conduite acceptable lorsqu'il s'agit d'affronter le monde surnaturel réellement, c'est-à-dire en dehors de la narration du mythe. Ainsi les chants sacrés ne contiennent-ils aucune référence à l'audace, à la supercherie et à la circonspection. Ce sont la politesse et la déférence qui caractérisent les invocations chantées aux divinités nommées, alors que les paroles à l'adresse d'êtres non nommés ont, comme je l'ai déjà dit, un caractère direct et assuré.

Les honneurs qu'un individu rend à un autre qui est socialement son supérieur se trouvent rehaussés par le rang du premier. Le mode d'interprétation des chants mis en danse par un groupe dont tous les membres sont de haut rang confirme cette identité sociale différée et contribue ainsi à sa permanence. Dans le cas d'un chant exaltant le rang d'un individu qui ne fait pas partie du groupe des danseurs, ces derniers sacrifient publiquement une part de leur individualité, par exemple en exécutant des mouvements non individualisés, c'est-à-dire synchronisés, et en portant le même costume. Ils s'effacent donc réellement pour la durée de la performance, tout en confirmant la supériorité du personnage dont le rang social complète le leur.

Ces ensembles de pouvoirs reflètent à leur tour les conceptions locales du pouvoir dans la nature et dans la société. Les représentations relatives à l'agencement des statuts sont ainsi centrées sur les attentes liées à l'efficacité appréciable ou à la capacité d'exercer un contrôle sur les pouvoirs (Goldman 1970: 8-9). Le pouvoir social est conçu comme une interaction, comme une sorte de contrepoint, entre le rang et les prérogatives qu'il confère; posséder l'un sans les autres, ou vice-versa, crée un déséquilibre dans le champ de tension entre le protocole et l'improvisation, entre ce qui est acquis et ce qui est attribué, entre le sacré et le profane, ou encore entre la sphère cérémonielle et la sphère pratique. Mais le rapport que le pouvoir social entretient avec la dichotomie sacré/profane n'est pas toujours mutuellement exclusif: l'agencement des rangs peut être modifié par l'intermédiaire des êtres surnaturels; d'un autre côté, les prêtres dépendent pour leur survie économique d'un patronage profane. L'une des conséquences du dynamisme de la culture polynésienne est qu'à certains moments historiques, les communautés de nombreux archipels élargissaient à l'univers entier les principes gouvernant la mise en œuvre et l'exercice du pouvoir social (voir Sahlins 1985: 215; Kaeppler 1985: 127). A long terme, la société oscillait ainsi entre une confusion et une séparation des sphères sociale et religieuse. De cette manière, les liens d'interdépendance qui unissaient les êtres 
humains à leurs univers naturel, social et surnaturel subissaient déjà des changements cumulatifs allant dans le sens d'une mutation culturelle - et ce indépendamment des transformations radicales engendrées plus tard par l'influence européenne (Leach 1984: 100).

Les deux fonctions principales remplies par la musique reflètent ce dualisme implicite des pouvoirs, comme le montre le schéma suivant:

\section{Changement de statut}

- importance de la créativité poétique

- individualité bienvenue

- origine récente et connue

- compositeur connu

- créativité verbale bienvenue

- style poétique marqué par des allusions et références indirectes

- créativité musicale désirée

- valorisation de la créativité

- performance publique généralement associée à la danse

- interprètes provenant du public

- performance symbolisant les structures sociales existantes

\section{Changement d'état}

- importance de l'adhésion au texte ancien

- individualité masquée par le costume, le rituel

- origine inconnue ou située dans le passé mythologique

- compositeur inconnu

- application rigide du texte fixe

- style poétique avec des références directes

- valorisation de l'adhésion à la mélodie ancienne ou au rythme ancien

- valorisation de la stabilité

- performance privée ou secrète rarement associée à la danse

- interprètes provenant de la prêtrise

- performance mobilisant les pouvoirs existants

La musique polynésienne joue donc sur deux plans opposés : elle est à la fois un facteur du dynamisme social et la garante de la stabilité culturelle. En franchissant les tensions et les contraintes porteuses de conflits, tels qu'ils caractérisent l'ensemble des sociétés polynésiennes, la musique porte la parole énoncée à un niveau quasi-mystique et touche ainsi d'une manière singulière le cœur des êtres humains autant que divins. Dans toutes ses manifestations, elle apparaît comme un élément essentiel du mouvement pendulaire du devenir social et culturel, qui retarde, maintient ou accélère le rythme du changement. 


\section{Bibliographie}

GOLDMAN Irving

1970 Ancient Polynesian Society. Chicago \& London: University of Chicago Press.

HIROA Te Rangi [Sir Peter Buck]

1966 The Coming of the Maori. Wellington: Whitcombe \& Tombs.

KAEPPLER Adrienne L.

1985 «Hawaian art and society». In: Antony Hooper \& Judith Huntsman (eds.), Transformations of Polynesian Culture. Auckland: Polynesian Society, p. 105-31.

LEACH Edmund

1984 «Oceans of opportunity?». In: Formal Proceedings: XV Pacific Science Congress, Dundein 1983. Dundein: Royal Society of New Zealand, p. 97-103.

McLEAN Mervyn \& Margaret ORBELL

1979 Traditional Songs of the Maori. Auckland: Auckland University Press \& Oxford University Press.

\section{MERRIAN Alan P.}

1964 The Anthropology of Music. Evanston, Ill.: Northwestern University Press.

MOYLE Richard M.

1988 Traditional Samoan Music. Auckland: Auckland University Press.

\section{ROBERTS Helen P.}

1926 Ancien Hawaiian Music. Honolulu: Bishop Museum (Bulletin 29).

SAHLINS Marshall

1985 «Hierarchy and humanity in Polynesia». In: Antony Hooper \& Judith Huntsman (eds.) Transformations of Polynesian Culture. Auckland: Polynesian Society, p. 195-217. 